\title{
Anomalous Right Coronary Artery Originating from the pulmo- nary artery. (ARCAPA): A systematic review of literature
} $\mathrm{H}_{\text {Rawal }}{ }^{*}$, SS Mehta ${ }^{2}$

University of Illinois at Urbana Champaign. 611 west Park Street, Urbana, Illinois 61801, USA

${ }^{2}$ Carle Foundation Hospital. Senior clinical and Interventional cardiologist. 611 west Park Street, Urbana, Illinois 61801 , USA

Article Info

\section{Article Notes}

Received: May 24, 2018

Accepted: June 13, 2018

\section{*Correspondence:}

Dr. H Rawal, University of Illinois at Urbana Champaign. 611 west Park Street, Urbana, Illinois 61801, USA; Email: harshrawal1210@yahoo.com.

C 2018 Rawal $\mathrm{H}$. This article is distributed under the terms of the Creative Commons Attribution 4.0 International License.

\section{ABSTRACT}

Anomalous origin of the right coronary artery from the pulmonary artery (ARCAPA) is a very rare congenital heart defect. Most patients are asymptomatic and the anomaly is detected incidentally during evaluation for other problems. Occasionally, ARCAPA may lead to myocardial ischemia and/or sudden cardiac arrest. Here we highlight the presentation, diagnosis and available treatment modalities for the management of this rare anomaly.

\section{Introduction}

Anomalous origin of the right coronary artery from the pulmonary artery (ARCAPA) is a rare congenital coronary anomaly. The incidence of ARCAPA is reported to be $0.002 \%$ in the literature and represents $0.12 \%$ of coronary anomalies ${ }^{1}$. Although more frequent in younger population, some patients have gone undiagnosed till the middle age where it could be diagnosed as an incidental finding. Compared to ARCAPA, Anomalous origin of the left coronary artery from pulmonary artery (ALCAPA) is usually discovered early on in life since these patients are usually symptomatic ${ }^{2}$. ARCAPA could exist by itself or in association with congenital heart diseases like tetralogy of fallot and bicuspid aortic valve ${ }^{3}$. The timeline for symptoms is variable in patients with ARCAPA and is related to the completeness of collateral circulation. Since a good number of patients remain asymptomatic the incidence may be higher compared to what has been reported ${ }^{4}$. Early symptoms may be secondary to heart failure, valvular insufficiencies or myocardial infarction ${ }^{5}$. Anomalous origin of coronary arteries typically from the contralateral sinus of valsalva (in particular the left coming off the right sinus) was more common compared to anomalous origin of right or left coronary arteries from the PA. These anomalies were more frequently associated with cause of sudden cardiac death in athletes in the USA ${ }^{6}$.

The pathophysiology of ARCAPA depends on the direction of blood flow in the coronary artery and its influence on oxygen delivery to the myocardium as well as the dominance. Patients with right dominant coronary circulation do not tolerate ARCAPA well compared with those with a left dominant system ${ }^{7}$. Coronary steal phenomenon can occur in ARCAPA due to differences in diastolic pressure between systemic and pulmonary arterial beds, with risk of myocardial ischemia during increased myocardial oxygen demand ${ }^{8}$. The presentation of ARCAPA is non-uniform and symptoms vary 
from effort angina to dyspnea or fatigue; however, in adulthood, ARCAPA could sometimes manifest with atypical chest pain or other nonspecific instrumental signs, such cardiac biomarker elevation suggestive for ischemic myocardial damage or arrhythmias (premature ventricular contractions to malignant ventricular tachycardia), both potential responsible for sudden cardiac death. Rarely, in infancy, heart failure cases have been reported which after further evaluation have turned out positive for ARCAPA $^{9}$. EKG in ARCAPA may be normal or it may show left ventricular hypertrophy or deep Q-waves in the inferior leads. Chest X-ray may vary from looking normal to showing signs of left ventricular hypertrophy.

\section{Diagnostic Imaging}

The first case of ARCAPA diagnosed by echocardiography was reported in $1885^{10}$. Nowadays, transthoracic echocardiogram (TTE) remains the common initial step to evaluate cardiac abnormalities. It can help delineate both coronary ostia, and doppler can provide an anatomic definition of proximal intramural course of the anomalous vessel. Although helpful transthoracic echocardiogram (TTE) is not always straightforward in adult patients as it is easier to visualize coronary ostia in infants and young children compared to adults. Color flow Doppler demonstrating intracoronary collaterals within the ventricular septum was thought to be highly indicative of ARCAPA ${ }^{11}$. Echocardiogram also shows dilated coronary arteries and a retrograde flow from the right coronary artery to the pulmonary artery suggestive of a normal phenomenon given the higher pressure in the RCA system thus creating a left to right shunt. However, in cases where TTE is difficult due to poor acoustic windows given the patient size, Transesophageal echocardiogram (TEE) could be an alternative first line diagnostic approach, providing more accurate details in suspicious of anomalous coronary takeoff and proximal course. Better visual confirmation can be obtained with coronary angiography, which most commonly show multiple collaterals from the left anterior descending artery (LAD) and left circumflex artery (LCX) to the RCA eventually draining into the pulmonary artery. Oximetry can be performed to see the level of left to right shunting in these patients where typically there is a step up of oxygenation from the right ventricle to the pulmonary artery.

More advanced imaging modalities like cardiac MRI (CMR) and CT angiography (CTA) can confirm previous echocardiographic findings. CMR provides with blood flow measurements by phase-contrast MRI per minute and per body surface area. Qp/Qs, which indicates pulmonary to systemic blood flow ratio, can be calculated using CMR. Besides assisting in quantifying the flow difference in assessment of the shunt, it can also assist with evaluation of right ventricular size and function, stress perfusion defects and possible myocardial fibrosis ${ }^{3}$. In centers without CMR, CTA can serve as a great alternative for diagnosis of ARCAPA. It additionally provides 3D volume rendering reconstructions for adequate images ${ }^{12}$.

\section{Management}

Treatment should be considered for patients presenting with symptoms before 35 years of age. Since the risk of sudden death decreases with age, symptomatic relief is the main goal for treatment ${ }^{13}$. Therapeutically, different approaches have been suggested and tried. Surgical techniques including the simple ligation of the RCA with saphenous vein bypass grafting and then reimplantation of the RCA into the aorta (Takeuchi procedure) is occasionally performed in children. The alternative method practiced was direct implantation of the RCA into the ascending aorta if possible ${ }^{13}$. Another alternative, usually in children, is the creation of a aortopulmonary window and establishing flow between the anomalous right coronary ostium and aorta using an intrapulmonary baffle or tunnel ${ }^{14}$.

Reported complication of thrombosis has been of concern in implanted coronary arteries. There have been three reported cases with complications of thrombosis after surgical correction of ARCAPA. Where the exact mechanism is not quite known, competitive flow including high and low flow states have been thought to be the likely explanations behind the thrombosis. Traditionally for such complications warfarin has been tried however, direct oral anticoagulants have not yet been tested or approved in this subgroup of patients. Given the low incidence and infrequent detection of these cases, larger studies are difficult to study the side effect profile of these new medications.

\section{Conclusion}

In conclusion, awareness amongst clinicians is important to make an early diagnosis of this rare anomaly. High suspicion is warranted in patients with multiple color Doppler signals detected across interventricular septum on echocardiographic evaluation. Atresia of the ostium of the RCA is a close differential of the ARCPA and can be associated with a dilated left coronary artery 9 . Echocardiogram (TTE,TEE) and coronary angiography are the common traditional methods for ARCAPA diagnosis; however, In light of advancing technology and current availability of other imaging modalities, earlier use of such resources can help making the diagnosis. Surgery remains the standard mode of correction of the anomaly and the method of correction is usually based on the surgeon's preference and experience.

\section{Conflict of interest disclosures}

The authors declare that there is no conflict of interest regarding the publication of this paper. 


\section{References}

1. Williams IA, Gersony WM, Hellenbrand WE. Anomalous right coronary artery arising from the pulmonary artery: a report of 7 cases and a review of the literature. Am Heart J. 2006; 152: 1004-1009.

2. Guzeltas A, Ozturk E, Tanidir IC, et al. "Evaluation of anomalous coronary arteries from the pulmonary artery," Brazilian Journal of Cardiovascular Surgery. 2017; vol. 32, no. 1: pp. 29-37.

3. Shariat M, Grosse-Wortmann L, Seed M, et al. "Isolated anomalous origin of the right coronary artery from the pulmonary artery in an asymptomatic 12-year-old girl: role of M RI in depicting the anatomy, detecting the ischemic burden, and quantifying the amount of leftto-right shunt,". World Journal for Pediatric and Congenital Heart Surgery. 2013; vol. 4, no. 2: pp. 201-205.Congenital Heart Surgery, vol. 4 , no. 2, pp. 201-205, 2013.

4. Balakrishna P, Illovsky M, Al-Saghir YM, et al. Anomalous Origin of Right Coronary Artery Originating from the Pulmonary Trunk (ARCAPA): an Incidental Finding in a Patient Presenting with Chest Pain. Cureus. 2017; 9(4): e1172.

5. Rodriguez-GonzalezM, TiradoAM, HosseinpourR, SotoJS. Anomalous origin of the left coronary artery from the pulmonary artery: diagnoses and surgical results in 12 pediatric patients. Tex Heart Inst J. 2015; 42(4): 350-6.

6. Maron BJ, Thompson PD, Puffer JC, et al. Cardiovascular preparticipation screening of competitive athletes. A statement for health professionals from the Sudden Death Committee (clinical cardiology) and Congenital Cardiac Defects Committee (cardio- vascular disease in the young), American Heart Association. Circulation. 1996; 94(4): 850-856.
7. Williams IA, Gersony WM, Hellenbrand WE. Anomalous right coronary artery arising from the pulmonary artery: A report of 7 cases and a review of the literature. Am Heart J. 2006; 152: 1004.e9-1004.e17.

8. Mehta SS, Sattiraju S. Multimodality Imaging of Anomalous Origin of Right Coronary Artery From Pulmonary Artery (ARCAPA). INVASIVE CARDIOL. 2017; 29(9): E104.

9. Sezen Ugan Atik, Levent Saltik, Kazim ozatarhan, et al. An incidentally detected anomalous origin of the right coronary artery from the pulmonary artery. Arch Argent Pediatr. 2018; 116(1): e102-e105 / e102.

10. Brooks HSJ. Two cases of an abnormal coronary artery of the heart arising from the pulmonary artery with some remarks upon the effect of this anomaly in producing cirsoid dilatation of the vessels. J Anat Physiol. 1885; 20 : 26-9.

11. KühnA, Kasnar-SamprecJ, SchreiberC, etal. Anomalous origin of the right coronary artery from the pulmonary artery (ARCAPA). Int J Cardiol. 2010; 139(2): e27-8.

12. Anders Svensson Raquel Themudo Kerstin Cederlund. Anomalous origin of right coronary artery from the pulmonary artery. European Heart Journal. 1 November 2017; Volume 38, Issue 41: Pages 3069.

13. .Kautzner J, Veselka J, Rohac J. Anomalous origin of the right coronary artery from the pulmonary trunk: is surgical reimplantation into the aorta a method of choice. Clin Cardiol. 1996; 19(3): 257-9.

14. Radke PW, Messmer BJ, Haager PK, et al. "Anomalous origin of the right coronary artery: preoperative and postoperative hemodynamics,". Annals of Thoracic Surgery. 1998; vol. 66, no. 4: pp. 1444-1449. 